\title{
EXACT SERIES RECONSTRUCTION IN PHOTOACOUSTIC TOMOGRAPHY WITH CIRCULAR INTEGRATING DETECTORS*
}

\author{
GERHARD ZANGERL ${ }^{\dagger}$, OTMAR SCHERZER ${ }^{\ddagger}$, AND MARKUS HALTMEIER ${ }^{\S}$
}

\begin{abstract}
A method for photoacoustic tomography is presented that uses circular integrals of the acoustic wave for the reconstruction of a three-dimensional image. Image reconstruction is a two-step process: In the first step data from a stack of circular integrating detectors are used to reconstruct the circular projection of the source distribution. In the second step the inverse circular Radon transform is applied. In this article we establish inversion formulas for the first step, which involves an inverse problem for the axially symmetric wave equation. Numerical results are presented that show the validity and robustness of the resulting algorithm.
\end{abstract}

Key words. Radon transform, photoacoustic tomography, photoacoustic microscopy, Hankel transform, image reconstruction, integrating detectors, axially symmetric, wave equation.

AMS subject classifications. 44A12, 65R32, 35L05, 92C55.

\section{Introduction}

The principle of Photoacoustic Tomography (PAT), also called Thermoacoustic tomography, is based on the excitation of high bandwidth acoustic waves with pulsed non-ionizing electromagnetic energy inside tissue [9, 17, 23, 25, 28]. PAT presents a hybrid imaging technique that combines the advantages of optical (high contrast) and ultrasound imaging (high resolution). It has proven great potential for important medical applications including cancer diagnostics $[16,21]$ and imaging of vasculature $[7,15]$.

The common approach uses small conventional piezoelectric transducers that approximate point detectors to measure the acoustic pressure [28]. Reconstruction algorithms which are based on the point detector assumption yield images with a spatial resolution that is essentially limited by the size of the used piezoelectric transducers [27]. The size of the detector can in principle be reduced, but only at the cost of also reducing the signal-to-noise ratio. In order to overcome this limitation large size planar or linear integrating detectors have been proposed in [3, 12]. Line shaped detectors integrate the acoustic pressure over its length and can be implemented by a Mach-Zehnder [22] or a Fabry-Perot interferometer [10], which naturally integrate the acoustic pressure over the length of a laser beam.

A drawback of linear integrating detectors is that because of attenuation parts of the detector which are distant from the object may be less influenced by the pressure wave. Moreover, linear integrating detectors do not provide a compact experimental buildup. In [32] so called circular integrating detectors where introduced, which in-

*Received: September 11, 2008; accepted (in revised version): June 15, 2009. Communicated by Lenya Ryzhik.

${ }^{\dagger}$ Department of Mathematics University of Innsbruck, Technikerstraße 21a, 6020 Innsbruck. Current address: Computational Science Center, University of Vienna, Nordbergstraße 15, 1090 Wien (Gerhard.Zangerl@univie.ac.at).

${ }^{\ddagger}$ Department of Mathematics University of Innsbruck, Technikerstraße 21a, 6020 Innsbruck, and Radon Institute of Computational and Applied Mathematics, Altenberger Strasße 69, 4040 Linz, Austria. Current address: Computational Science Center, University of Vienna, Nordbergstraße 15, 1090 Wien (Otmar.Scherzer@univie.ac.at).

$\S$ Department of Mathematics, University of Innsbruck, Technikerstraße 21a, 6020 Innsbruck. Current address: Computational Science Center, University of Vienna, Nordbergstraße 15, 1090 Wien (Markus.Haltmeier@univie.ac.at). 


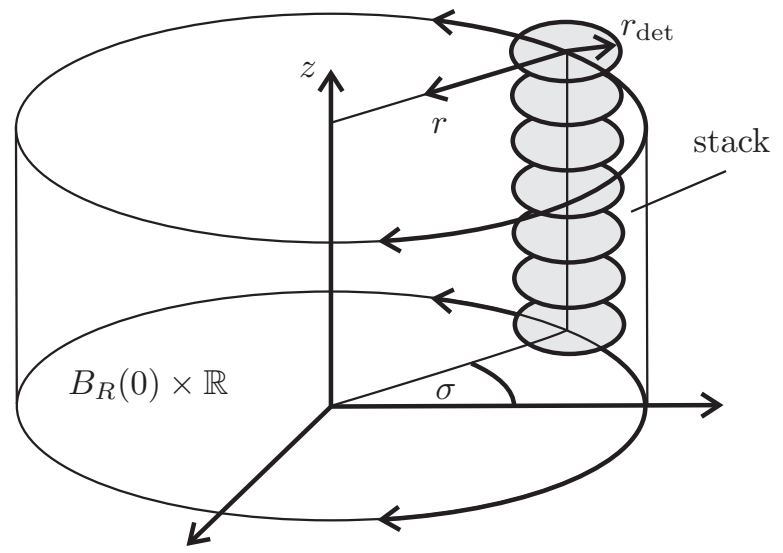

FIG. 1.1. Scanning geometry: a stack of circles centered on the boundary of $B_{R}(0) \times \mathbb{R}$ is rotated around the $\mathbf{e}_{3}$-axis.

tegrate the acoustic pressure over circles. A circular integrating detector can, similar to a linear integrating detector, be implemented by an interferometer where the laser beam is guided along a circle in an optical fiber. It is free of any aperture effect and can provide a uniformly and high resolution throughout the image area. Since it is possible to fabricate optical fibers out of materials which have nearly the same acoustical density like the fluid in which they are contained [10] no shadowing effects due to the circular integrating detectors are expected. The use of circular integrating detectors has been proposed independently in [31]. However, their study was limited to two spatial dimensions, where the circular shaped detector can be used as virtual point detector.

In [32] we derived an inversion formula based on the expansion of the involved functions in special basis-functions. This formula, is hard to implement directly due to possible division by a zero (see Subsection 2.2). In this article we prove two novel exact series solutions that allow for stable implementation. As a byproduct we obtain a novel inversion formula for PAT using point-like detectors on a cylindrical recording surface (see Remark 3.6).

The outline of this article is as follows. In section 2 we review PAT with circular integrating detectors and recall the main results of [32]. In the following sections we will be concerned with derivation and implementation of the novel stable inversion formulas.

\section{Photoacoustic tomography with circular integrating detectors}

In PAT an acoustic pressure wave $p(\mathbf{x}, t)$, inside an object, is generated by a pulse of non-ionizing electromagnetic radiation. In the case of spatially constant sound speed, the induced acoustic pressure satisfies the initial value problem [25, 28]

$$
\begin{aligned}
\partial_{t}^{2} p(\mathbf{x}, t) & =\triangle p(\mathbf{x}, t), & & (\mathbf{x}, t) \in \mathbb{R}^{3} \times(0, \infty), \\
p(\mathbf{x}, 0) & =f(\mathbf{x}), & & \mathbf{x} \in \mathbb{R}^{3}, \\
\partial_{t} p(\mathbf{x}, 0) & =0, & & \mathbf{x} \in \mathbb{R}^{3} .
\end{aligned}
$$

PAT is concerned with recovering the initial pressure from measurements of $p$ outside the support of $f$. 
In [32] it was proposed to measure the acoustic signals with a stack of parallel circles which is rotated around a single axis; see figure 1.1. In such a situation, threedimensional imaging involves the inversion of the classical circular Radon transform and the inversion of a reduced wave equation, as outlined in the following.

Throughout this article it is assumed that $f$ is smooth and supported in the cylinder $B_{R}(0) \times \mathbb{R}$, where $R$ is a fixed positive number. Let $p(x, t)$ denote the unique solution of (2.1)-(2.3) and, for $\sigma \in S^{1}$, define

$$
\begin{aligned}
P_{\sigma}(z, r, t):=\frac{1}{2 \pi} \int_{0}^{2 \pi} p\left(\boldsymbol{\Phi}_{\sigma}(z, r, \alpha), t\right) d \alpha, & & (z, r, t) \in \mathbb{R} \times(0, \infty)^{2}, \\
F_{\sigma}(z, r):=\frac{1}{2 \pi} \int_{0}^{2 \pi} f\left(\boldsymbol{\Phi}_{\sigma}(z, r, \alpha)\right) d \alpha, & & (z, r) \in \mathbb{R} \times(0, \infty),
\end{aligned}
$$

where

$$
\mathbf{\Phi}_{\sigma}(z, r, \alpha)=R \sigma+(r \cos (\alpha), r \sin (\alpha), z)^{T}, \quad(z, r, \alpha) \in \mathbb{R} \times(0, \infty) \times[0,2 \pi] .
$$

The stack of circular integrating detectors measures are

$$
G_{\sigma}(z, t):=P_{\sigma}\left(z, r_{\mathrm{det}}, t\right), \quad(\sigma, z, t) \in S^{1} \times \mathbb{R} \times(0, \infty),
$$

with $r_{\text {det }}>0$ denoting the fixed radius of the detectors.

The goal is to recover the unknown initial data $f$ from measured data $\left(G_{\sigma}\right)_{\sigma \in S^{1}}$.

2.1. Two stage reconstruction. Reconstruction with circular integrating detectors is based on the following reduction to the axial symmetric wave equation:

Proposition 2.1. Let $f \in C_{0}^{\infty}\left(B_{R}(0) \times \mathbb{R}\right)$ and define $P_{\sigma}$ and $F_{\sigma}, \sigma \in S^{1}$, by (2.4), (2.5). Then $P_{\sigma}$ satisfies the axial symmetric wave equation

$$
\begin{aligned}
\partial_{t}^{2} P_{\sigma}(z, r, t) & =\left(r^{-1} \partial_{r} r \partial_{r}+\partial_{z}^{2}\right) P_{\sigma}(z, r, t), & & (z, r, t) \in \mathbb{R} \times(0, \infty)^{2}, \\
P_{\sigma}(z, r, 0) & =F_{\sigma}(z, r), & & (z, r) \in \mathbb{R} \times(0, \infty), \\
\partial_{t} P_{\sigma}(z, r, 0) & =0, & & (z, r) \in \mathbb{R} \times(0, \infty) .
\end{aligned}
$$

Moreover $P_{\sigma}$ remains bounded as $r \rightarrow 0$.

Proof. Equations (2.8), (2.9) and the boundedness as $r \rightarrow 0$ immediately follow from (2.2), (2.3) and the definitions of $P_{\sigma}$ and $F_{\sigma}$. In the cylindrical coordinates $\boldsymbol{\Phi}_{\sigma}$, the Laplace operator is given by the well known expression

$$
\Delta=r^{-1} \partial_{r} r \partial_{r}+\partial_{z}^{2}+r^{-2} \partial_{\alpha}^{2} .
$$

Integrating the equation $\Delta p=\partial_{t}^{2} p$ with respect to $\alpha$ yields (2.7).

Note that (2.7)-(2.9) is uniquely solvable if we require that its solution remains bounded as $r \rightarrow 0$.

Remark 2.2. Proposition 2.1 is the basis of the following two-stage procedure which reconstructs the initial pressure $f$ in $(2.1)-(2.3)$ from the data $\left(G_{\sigma}\right)_{\sigma \in S^{1}}$ :

(i) For $\sigma \in S^{1}$ (fixed position of the stack of circles) determine the initial pressure $F_{\sigma}$ of (2.7)-(2.9) using data $G_{\sigma}$.

Repeating this procedure for every $\sigma$, one obtains a family of functions $F_{\sigma}$, $\sigma \in S^{1}$, corresponding to averages over circles centered on $\partial\left(B_{R}(0) \times \mathbb{R}\right)$. 
(ii) Next one recognizes that for fixed $z=z_{0}$, the function

$$
(\sigma, r) \mapsto F_{\sigma}\left(z_{0}, r\right)
$$

is the circular mean transform of $\left.f\right|_{\left\{z=z_{0}\right\}}$ with centers on a circle. For the circular mean transform stable analytic inversion formulas have been recently discovered $[2,8,11,18,19]$. Exemplarily, one of the formulas in $[8$, Theorem 1.1] reads

$$
f\left(\mathbf{x}^{\prime}, z_{0}\right)=\frac{1}{2 \pi} \int_{S^{1}}\left(\int_{0}^{2 R}\left(\partial_{r} r \partial_{r} F_{\sigma}\right)\left(z_{0}, r\right) \log \left|r^{2}-\right| \mathbf{x}^{\prime}-\left.R \sigma\right|^{2} \mid d r\right) d \sigma
$$

where $\mathbf{x}^{\prime}$ denotes the coordinates in the plane $\mathbb{R}^{2} \times\left\{z_{0}\right\}$.

The key task for reconstruction $f$ is to derive stable and fast algorithms to reconstruct the initial data in (2.7)-(2.9) from measurement data $G_{\sigma}$. A possible reconstruction method is based of time reversal (back-propagation) similar to $[4,5,14]$. However, the degeneration of $r^{-1} \partial_{r} r \partial_{r}$ at $r=0$ and the open detector set may cause difficulties in such procedures. The inversion approach in this paper is based on analytic inversion formulas for reconstructing $F_{\sigma}$.

2.2. Exact inversion formula. In the following denote by

$$
\begin{array}{ll}
\mathbf{S}\{\phi\}(\omega):=\int_{0}^{\infty} \phi(t) \sin (\omega t) d t, & \phi \in L^{1}((0, \infty)), \omega>0, \\
\mathbf{C}\{\phi\}(\omega):=\int_{0}^{\infty} \phi(t) \cos (\omega t) d t, & \phi \in L^{1}((0, \infty)), \omega>0, \\
\mathbf{F}\{\phi\}(k):=\int_{\mathbb{R}} \phi(z) e^{-i k z} d z, & \phi \in L^{1}(\mathbb{R}), k \in \mathbb{R}, \\
\mathbf{H}\{\phi\}(v):=\int_{0}^{\infty} \phi(r) J_{0}(v r) r d r, & \phi \in L^{1}\left((0, \infty), r^{1 / 2} d r\right), v>0,
\end{array}
$$

the sine, cosine, Fourier, and Hankel transform, respectively. (Here $J_{0}$ is the zero order Bessel function [1].) When the above transforms are applied to functions depending on several variables then the transformed variable is added as subscript, e.g., $\mathbf{H}_{r}\left\{F_{\sigma}\right\}(z, v)=\int_{0}^{\infty} F_{\sigma}(z, r) J_{0}(v r) r d r$.

Proposition 2.3. Let $f \in C_{0}^{\infty}\left(B_{R}(0) \times \mathbb{R}\right)$ and define $F_{\sigma}(z, r)$ and $G_{\sigma}(z, t)$ by (2.5), (2.6). Then the relation

$$
\mathbf{H}_{r}\left\{\mathbf{F}_{z}\left\{F_{\sigma}\right\}\right\}(k, v)=\frac{2}{\pi} \frac{\mathbf{C}_{t}\left\{\mathbf{F}_{z}\left\{G_{\sigma}\right\}\right\}\left(k, \sqrt{k^{2}+v^{2}}\right)}{J_{0}\left(r_{\operatorname{det}} v\right) \sqrt{k^{2}+v^{2}}}
$$

holds whenever $J_{0}\left(r_{\operatorname{det}} v\right) \neq 0$.

Proof. The zero order Bessel function satisfies $r^{-1} \partial_{r} r \partial_{r} J_{0}(r)=-J_{0}(r)$ on $(0, \infty)$. Hence the chain rule implies that $r^{-1} \partial_{r} r \partial_{r} J_{0}(r v)=-v^{2} J_{0}(r v)$ for every $r, v>0$. Separation of variables shows that the functions

$$
(z, r, t) \mapsto e^{i k z} \cos \left(t \sqrt{k^{2}+v^{2}}\right) J_{0}(r v), \quad(k, v) \in \mathbb{R} \times(0, \infty),
$$

solve (2.7), (2.9). Employing the initial condition (2.8) and the inversion formulas for the Fourier and Hankel transforms it follows that the unique bounded solution of 
(2.7)-(2.9) is given by

$$
P_{\sigma}(r, z, t):=\frac{1}{2 \pi} \int_{\mathbb{R}} \int_{0}^{\infty} \bar{F}(k, v) e^{i k z} J_{0}(r v) \cos \left(t \sqrt{k^{2}+v^{2}}\right) v d v d k,
$$

with $\bar{F}(k, v):=\mathbf{H}_{r}\left\{\mathbf{F}_{z}\left\{F_{\sigma}\right\}\right\}(k, v)$.

Substituting $\omega=\sqrt{k^{2}+v^{2}}$ in (2.12) and putting $r=r_{\text {det }}$ afterwards leads to

$$
G_{\sigma}(z, t)=\frac{1}{2 \pi} \int_{\mathbb{R}}\left(\int_{|k|}^{\infty} J_{0}\left(r_{\operatorname{det}} \sqrt{\omega^{2}-k^{2}}\right) \omega \bar{F}\left(k, \sqrt{\omega^{2}-k^{2}}\right) \cos (\omega t) d \omega\right) e^{i k z} d k
$$

The inversion formulas for the Fourier and Cosine transforms now imply that

$$
\mathbf{C}_{t}\left\{\mathbf{F}_{z}\left\{G_{\sigma}\right\}\right\}(k, \omega)=\frac{\pi}{2} \begin{cases}J_{0}\left(r_{\operatorname{det}} \sqrt{\omega^{2}-k^{2}}\right) \omega \bar{F}\left(k, \sqrt{\omega^{2}-k^{2}}\right), & \text { if } \omega>k, \\ 0, & \text { otherwise. }\end{cases}
$$

Solving the last equation for $\bar{F}$ shows (2.11).

Proposition 2.3 implies that $F_{\sigma}$ can be reconstructed from data $G_{\sigma}$ as follows:

(i) The data $G_{\sigma}$ are Fourier and cosine transformed, yielding to $\mathbf{C}_{t}\left\{\mathbf{F}_{z}\left\{G_{\sigma}\right\}\right\}$.

(ii) According to (2.11), $\mathbf{C}_{t}\left\{\mathbf{F}_{z}\left\{G_{\sigma}\right\}\right\}$ is mapped to $\mathbf{H}_{r}\left\{\mathbf{F}_{z}\left\{F_{\sigma}\right\}\right\}$.

(iii) Finally, application of the inverse Fourier and Hankel transforms yields

$$
F_{\sigma}(z, r)=\frac{1}{2 \pi} \int_{\mathbb{R}} \int_{0}^{\infty} \mathbf{H}_{r}\left\{\mathbf{F}_{z}\left\{F_{\sigma}\right\}\right\}(k, v) J_{0}(r v) e^{i k z} v d v d k .
$$

REMARK 2.4 (Instability of (2.11)). Inversion formula (2.11) is not defined when $J_{0}\left(r_{\operatorname{det}} v\right)$ equals 0 . From the proof of the above theorem it is clear that for exact data

$$
\mathbf{C}_{t}\left\{\mathbf{F}_{z}\left\{G_{\sigma}\right\}\right\}\left(k, \sqrt{v_{n}^{2}+k^{2}}\right)=0, \quad n \in \mathbb{N},
$$

with $\left(v_{n}\right)_{n \in \mathbb{N}}$ denoting the zeros of $r \mapsto J_{0}\left(r_{\operatorname{det}} v\right)$. In practice, however, only noisy (approximately measured) data $G_{\sigma}^{\delta} \simeq G_{\sigma}$ are available. In general,

$$
\mathbf{C}_{t}\left\{\mathbf{F}_{z}\left\{G_{\sigma}^{\delta}\right\}\right\}\left(k, \sqrt{v_{n}^{2}+k^{2}}\right) \neq 0 .
$$

It is therefore difficult to stably evaluate the quotient in (2.11) in practice.

Because the acoustic pressure is measured outside of the investigated object, only the following two situations occur in practical applications, see figure 2.1:

(i) The stack of circles is strictly outside the object. In this case $r_{\text {det }}<R$ and $\operatorname{supp}(f) \subset B_{R-r_{\mathrm{det}}}(0) \times \mathbb{R}$.

(ii) The object is enclosed in the stack of circles. In this case $r_{\text {det }} \geq 2 R$.

For the case $r_{\text {det }} \geq 2 R$ we will provide two stable formulas based on expansions in bases of special functions. The case $r_{\text {det }}<R$ turns out to be harder, we currently do not have a stable alternative to $(2.11)$. In this case the function $\mathbf{F}\left\{F_{\sigma}\right\}(k, \cdot)$ is not supported in the interval $\left(0, r_{\mathrm{det}}\right)$ and thus it cannot be expanded into a Fourier Bessel series which is crucial in the proofs of Theorems 3.1 and 3.4. However, in the limiting case $r_{\text {det }} \ll R$ a stable reconstruction formula is obtained; compare with Remark 3.6 below. 

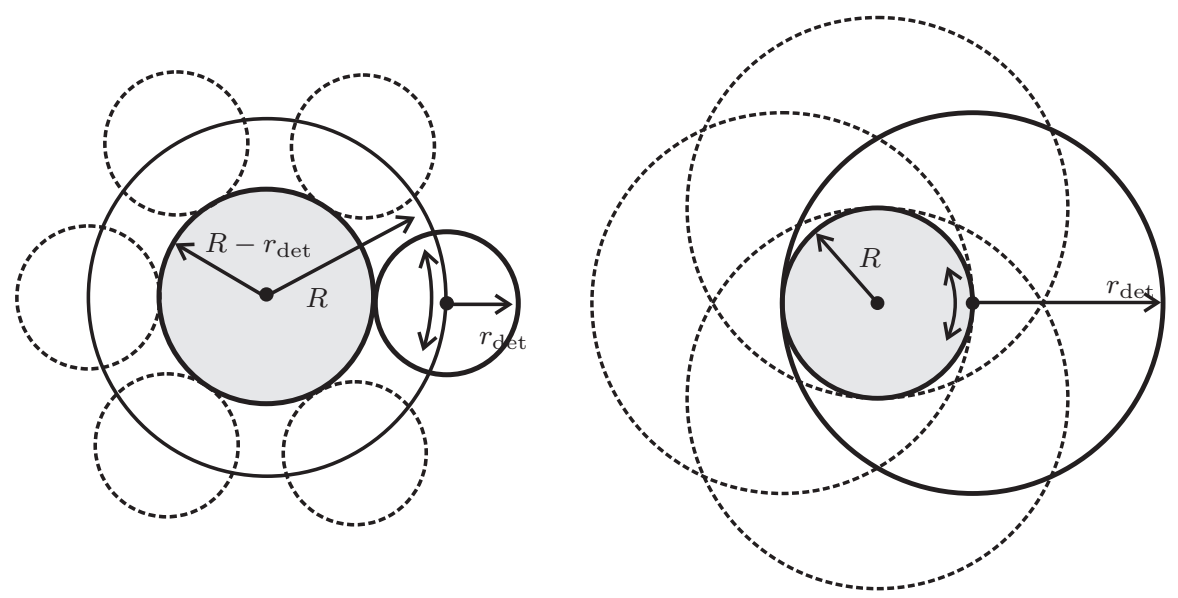

FIG. 2.1. Cross section of experimental buildup when $r_{\mathrm{det}}<R$ (left) and $r_{\mathrm{det}} \geq 2 R$ (right). In both case object has to be supported in the gray disc.

\section{Stable inversion formulas}

In the following we fix $f \in C_{0}^{\infty}\left(B_{R}(0) \times \mathbb{R}\right), \sigma \in S^{1}$, define $F_{\sigma}, G_{\sigma}$ by (2.5), (2.6), and let $\left(v_{n}\right)_{n \in \mathbb{N}}$ denote the zeros of the function $v \mapsto J_{0}\left(r_{\operatorname{det}} v\right)$.

Our first stable inversion formula is as follows:

Theorem 3.1 (The L'Hospital trick). Assume $r_{\text {det }} \geq 2 R$. Then

$$
F_{\sigma}(z, r)=\frac{2}{\pi^{2} r_{\operatorname{det}}^{3}} \int_{\mathbb{R}}\left(\sum_{n \in \mathbb{N}} \frac{\mathbf{S}_{t}\left\{t \mathbf{F}_{z}\left\{G_{\sigma}\right\}\right\}\left(k, \sqrt{k^{2}+v_{n}^{2}}\right) v_{n}}{k^{2}+v_{n}^{2}} \frac{J_{0}\left(r v_{n}\right)}{J_{1}\left(r_{\operatorname{det}} v_{n}\right)^{3}}\right) e^{i k z} d k
$$

for any $(z, r) \in \mathbb{R} \times(0, \infty)$.

Proof. The assumptions $f \in C_{0}^{\infty}\left(B_{R}(0) \times \mathbb{R}\right)$ and $r_{\operatorname{det}} \geq 2 R$ imply that $\mathbf{F}_{z}\left\{F_{\sigma}\right\}(k, \cdot)$ is compactly supported in $\left(0, r_{\text {det }}\right)$. It can therefore be expanded in a Fourier Bessel series [26]

$$
\mathbf{F}_{z}\left\{F_{\sigma}\right\}(k, r)=\frac{2}{r_{\operatorname{det}}^{2}} \sum_{n \in \mathbb{N}} \mathbf{H}_{r}\left\{\mathbf{F}_{z}\left\{F_{\sigma}\right\}\right\}\left(k, v_{n}\right) \frac{J_{0}\left(r v_{n}\right)}{J_{1}\left(r_{\operatorname{det}} v_{n}\right)^{2}} .
$$

According to (2.11) we have

$$
\mathbf{H}_{r}\left\{\mathbf{F}_{z}\left\{F_{\sigma}\right\}\right\}(k, v)=\frac{2}{\pi} \frac{\mathbf{C}_{t}\left\{\mathbf{F}_{z}\left\{G_{\sigma}\right\}\right\}\left(k, \sqrt{k^{2}+v^{2}}\right)}{J_{0}\left(r_{\operatorname{det}} v\right) \sqrt{k^{2}+v^{2}}}, \quad v \notin\left\{v_{n}: n \in \mathbb{N}\right\} .
$$

Applying the rule of L'Hospital gives

$$
\begin{aligned}
\mathbf{H}_{r}\left\{\mathbf{F}_{z}\left\{F_{\sigma}\right\}\right\}\left(k, v_{n}\right) & =\frac{2}{\pi} \lim _{v \rightarrow v_{n}} \frac{\partial / \partial v\left[\mathbf{C}_{t}\left\{\mathbf{F}_{z}\left\{G_{\sigma}\right\}\right\}\left(k, \sqrt{k^{2}+v^{2}}\right)\right]}{\partial / \partial v\left[J_{0}\left(r_{\operatorname{det}} v\right) \sqrt{k^{2}+v^{2}}\right]} \\
& =\frac{2}{\pi} \lim _{v \rightarrow v_{n}} \frac{\mathbf{S}_{t}\left\{t \mathbf{F}_{z}\left\{G_{\sigma}\right\}\right\}\left(k, \sqrt{k^{2}+v^{2}}\right) \frac{v}{\sqrt{k^{2}+v^{2}}}}{J_{1}\left(r_{\operatorname{det}} v\right) r_{\operatorname{det}} \sqrt{k^{2}+v^{2}}} \\
& =\frac{2}{\pi r_{\operatorname{det}}} \frac{\mathbf{S}_{t}\left\{t \mathbf{F}_{z}\left\{G_{\sigma}\right\}\right\}\left(k, \sqrt{k^{2}+v_{n}^{2}}\right) v_{n}}{J_{1}\left(r_{\operatorname{det}} v_{n}\right)\left(k^{2}+v_{n}^{2}\right)} .
\end{aligned}
$$


Inserting (3.3) in (3.2) and using the Fourier inversion formula shows (3.1).

REMARK 3.2 (Stability of (3.1)). From the asymptotic approximation (see [1])

$$
J_{m}(x) \simeq \sqrt{\frac{2}{\pi x}} \cos \left(x-\frac{m \pi}{2}-\frac{\pi}{4}\right), \quad \text { for } x \rightarrow \infty,
$$

of the $m$-th order Bessel function it follows that

$$
v_{n} \simeq \frac{\pi(n+1 / 4)}{r_{\operatorname{det}}}, \quad\left|J_{1}\left(r_{\operatorname{det}} v_{n}\right)\right| \simeq \sqrt{\frac{2}{\pi r_{\operatorname{det}} v_{n}}}, \quad \text { for } n \rightarrow \infty .
$$

Moreover the summands in (3.1) take the asymptotic form

$$
\begin{aligned}
\mid \mathbf{S}_{t}\{t & \left.\mathbf{F}_{z}\left\{G_{\sigma}\right\}\right\}\left(k, \sqrt{k^{2}+v_{n}^{2}}\right) \frac{v_{n}}{k^{2}+v_{n}^{2}} \frac{J_{0}\left(r v_{n}\right)}{J_{1}\left(r_{\operatorname{det}} v_{n}\right)^{3}} \mid \\
& \simeq\left|\mathbf{S}_{t}\left\{t \mathbf{F}_{z}\left\{G_{\sigma}\right\}\right\}\left(k, \sqrt{k^{2}+v_{n}^{2}}\right)\right| \frac{1}{v_{n}} \frac{\left|\cos \left(v_{n}-\frac{\pi}{4}\right)\right|\left(2 /\left(\pi r v_{n}\right)\right)^{1 / 2}}{\left(2 /\left(\pi r_{\operatorname{det}} v_{n}\right)\right)^{3 / 2}} \\
& \leq \frac{r^{1 / 2}}{4 r_{\text {det }}^{3 / 2}}\left|\mathbf{S}_{t}\left\{t \mathbf{F}_{z}\left\{G_{\sigma}\right\}\right\}\left(k, \sqrt{k^{2}+v_{n}^{2}}\right)\right| .
\end{aligned}
$$

Consequently, the parts that do not depend on the data $G_{\sigma}$ are bounded, and (3.1) can be implemented in stable way.

In the sequel we derive an additional inversion formula that circumvents the division by zero problem. In fact, our formula will be a consequence of the following result derived in [30].

Proposition 3.3. Let $p$ denote the unique solution of (2.1)-(2.3) and let $f_{\sigma}^{m}$ and $g_{\sigma}^{m}$ denote the Fourier coefficients of $f\left(\mathbf{\Phi}_{\sigma}(z, r, \alpha)\right)$ and

$$
g_{\sigma}(\alpha, z, t):= \begin{cases}p\left(\boldsymbol{\Phi}_{\sigma}(z, r, \alpha), t\right), & \text { for } t>0, \\ 0, & \text { otherwise, }\end{cases}
$$

with respect to $\alpha$. Then

$$
\mathbf{H}_{r}\left\{\mathbf{F}_{t}\left\{f_{\sigma}^{m}\right\}\right\}(k, v)=\frac{2}{\pi} \frac{\mathbf{F}_{t}\left\{\mathbf{F}_{z}\left\{g_{\sigma}^{m}\right\}\right\}\left(k, \sqrt{k^{2}+v^{2}}\right)}{H_{m}^{(2)}\left(r_{\operatorname{det}} v\right) \sqrt{k^{2}+v^{2}}}, \quad(k, v) \in \mathbb{R} \times(0, \infty),
$$

with $H_{m}^{(2)}$ denoting the $m$-th order second kind Hankel function.

Now the second stable inversion formula can be stated as follows:

Theorem 3.4. Assume $r_{\mathrm{det}} \geq 2 R$. Then

$$
F_{\sigma}(z, r)=\frac{2}{\pi^{2} r_{\operatorname{det}}^{2}} \int_{\mathbb{R}}\left(\sum_{n \in \mathbb{N}} \frac{\mathbf{F}_{t}\left\{\mathbf{F}_{z}\left\{G_{\sigma}\right\}\right\}\left(k, \sqrt{k^{2}+v_{n}^{2}}\right)}{H_{0}^{(2)}\left(r_{\operatorname{det}} v_{n}\right) \sqrt{k^{2}+v_{n}^{2}}} \frac{J_{0}\left(r v_{n}\right)}{J_{1}\left(r_{\operatorname{det}} v_{n}\right)^{2}}\right) e^{i k z} d k .
$$

Here $G_{\sigma}$ is extended by $G_{\sigma}(z, t)=0$ for $t<0$.

Proof. We use again the Fourier-Bessel series (3.2) of proof of Theorem 3.1. Recalling the definitions of $F_{\sigma}, G_{\sigma}$ and the Fourier coefficients $f_{\sigma}^{m}, g_{\sigma}^{m}$ one notices that $F_{\sigma}=f_{\sigma}^{0}, G_{\sigma}=g_{\sigma}^{0}$. Therefore (3.4) for $m=0$ implies that

$$
\mathbf{H}_{r}\left\{\mathbf{F}_{z}\left\{F_{\sigma}\right\}\right\}(k, v)=\frac{2}{\pi} \frac{\mathbf{F}_{t}\left\{\mathbf{F}_{z}\left\{G_{\sigma}\right\}\right\}\left(k, \sqrt{k^{2}+v^{2}}\right)}{H_{0}^{(2)}\left(r_{\operatorname{det}} v\right) \sqrt{k^{2}+v^{2}}}, \quad(k, v) \in \mathbb{R} \times(0, \infty) .
$$


Inserting (3.6) in (3.2) and using the Fourier inversion formula shows (3.5).

Equation (3.6) is quite similar to (2.11). However, in the denominator in (3.6) the zero order second kind Hankel function appears (instead of the the zero order Bessel function) which cannot be zero for a finite argument [1]. Moreover, the asymptotic expansion of the Bessel and the second kind Hankel function show that the summands in (3.5) that do not depend on the data $G_{\sigma}$ remain bounded as $n \rightarrow \infty$.

REMARK 3.5. The derivation of (3.4) is based on the following Green's function expansion in cylindrical coordinates [30]

$$
\begin{aligned}
& \frac{e^{-i \omega\left|\mathbf{\Phi}_{\sigma}(z, r, \alpha)-\boldsymbol{\Phi}_{\sigma}\left(z_{0}, r_{\mathrm{det}}, \alpha_{0}\right)\right|}}{\left|\boldsymbol{\Phi}_{\sigma}(z, r, \alpha)-\boldsymbol{\Phi}_{\sigma}\left(z_{0}, r_{\mathrm{det}}, \alpha_{0}\right)\right|} \\
& =\frac{-i \pi}{2} \int_{\mathbb{R}}\left(\sum_{m \in \mathbb{Z}} A_{m}\left(v r, v r_{\mathrm{det}}\right) e^{-i m\left(\alpha-\alpha_{0}\right)}\right) e^{-i \omega\left(z-z_{0}\right)} d z,
\end{aligned}
$$

with $v=\operatorname{sign}(\omega) \sqrt{\left|\omega^{2}-k^{2}\right|}$,

$$
A_{m}\left(v r, v r_{\mathrm{det}}\right)= \begin{cases}H_{m}^{(2)}\left(v r_{\mathrm{det}}\right) J_{m}(v r), & \text { if } \omega^{2}>k^{2} \\ 2 i / \pi K_{m}\left(|v| r_{\mathrm{det}}\right) I_{m}(|v| r), & \text { otherwise }\end{cases}
$$

and $I_{m}, K_{m}$ denoting the $m$-th order modified Bessel functions of first and second kind, respectively. Here its is assumed that $r_{\text {det }}>r$.

Interchanging the roles of $r$ and $r_{\mathrm{det}}$ implies that for $r_{\mathrm{det}}>r$ the Green function expansion (3.7) holds with

$$
A_{m}\left(v r, v r_{\mathrm{det}}\right)=\left\{\begin{array}{lr}
J_{m}\left(v r_{\mathrm{det}}\right) H_{m}^{(2)}(v r), & \text { if } \omega^{2}>k^{2}, \\
2 i / \pi I_{m}\left(|v| r_{\mathrm{det}}\right) K_{m}(|v| r), & \text { otherwise }
\end{array}\right.
$$

Similar to the proof of (3.4) in [30] this leads to a formula for reconstructing $F_{\sigma}$ in the case $r_{\text {det }} \leq 2 R$, which is again unstable since $J_{0}\left(v r_{\text {det }}\right)$ appears in the denominator.
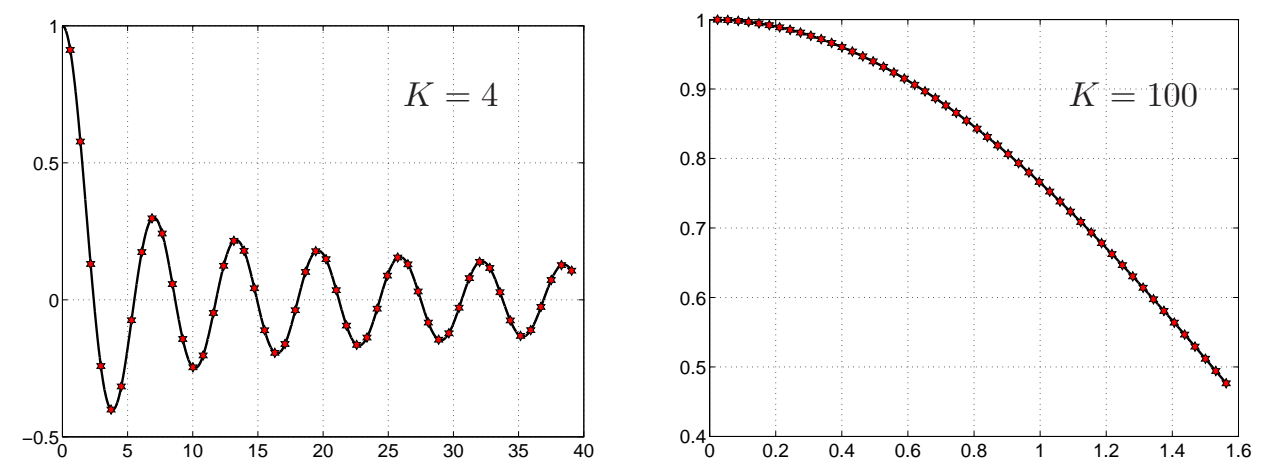

FIG. 3.1. The first 50 denominators $J_{0}\left(r_{\operatorname{det}} v_{n} / K\right)$ in (3.10) for $K=4$ (left) and $K=100$ (right).

Remark 3.6. Suppose that $r_{\operatorname{det}}<R$ and that $f$ is supported in $B_{R-r_{\operatorname{det}}}(0) \times \mathbb{R}$. For $r_{1} \geq 2 R-r_{\text {det }}$ let $\left(\tilde{v}_{n}\right)_{n \in \mathbb{N}}$ denote the zeros of $v \mapsto J_{0}\left(r_{1} \tilde{v}\right)$. Then one can expand 
$\mathbf{F}_{z}\left\{F_{\sigma}\right\}(k, \cdot)$ in a Fourier Bessel series (see [26])

$$
\mathbf{F}_{z}\left\{F_{\sigma}\right\}(k, r)=\frac{2}{r_{1}^{2}} \sum_{n \in \mathbb{N}} \mathbf{H}_{r}\left\{\mathbf{F}_{z}\left\{F_{\sigma}\right\}\right\}\left(k, \tilde{v}_{n}\right) \frac{J_{0}\left(r \tilde{v}_{n}\right)}{J_{1}\left(r_{1} \tilde{v}_{n}\right)^{2}}, \quad(k, r) \in \mathbb{R} \times(0, \infty) .
$$

According to (2.11) we have

$$
\mathbf{H}_{r}\left\{\mathbf{F}_{z}\left\{F_{\sigma}\right\}\right\}(k, v)=\frac{2}{\pi} \frac{\mathbf{C}_{t}\left\{\mathbf{F}_{z}\left\{G_{\sigma}\right\}\right\}\left(k, \sqrt{k^{2}+v^{2}}\right)}{J_{0}\left(r_{\operatorname{det}} v\right) \sqrt{k^{2}+v^{2}}} \quad v \notin\left\{v_{n}: n \in \mathbb{N}\right\} .
$$

If we assume that $r_{1}$ is a integer multiple of $r_{\text {det }}$, i.e., $r_{1}=K r_{\text {det }}$, then $\tilde{v}_{n}=v_{n} / K \notin$ $\left\{v_{m}: m \in \mathbb{N}\right\}$ for any $n \in \mathbb{N}$. Therefore, inserting (3.9) in (3.8) yields

$$
\mathbf{F}_{z}\left\{F_{\sigma}\right\}(k, r)=\frac{2}{\pi^{2} r_{1}^{2}} \sum_{n \in \mathbb{N}} \frac{\mathbf{C}_{t}\left\{\mathbf{F}_{z}\left\{G_{\sigma}\right\}\right\}\left(k, \sqrt{k^{2}+\tilde{v}_{n}^{2}}\right)}{J_{0}\left(r_{\operatorname{det}} \tilde{v}_{n}\right) \sqrt{k^{2}+\tilde{v}_{n}^{2}}} \frac{J_{0}\left(r \tilde{v}_{n}\right)}{J_{1}\left(r_{1} \tilde{v}_{n}\right)^{2}}
$$

In general, (3.10) is again sensitive to noise when $\tilde{v}_{n}$ gets close to a zero of $J_{0}\left(r_{\operatorname{det}} v\right)$.

In the limiting case $r_{\text {det }} \ll R$ and for $n$ not too large, the denominators $J_{0}\left(r_{1} \tilde{v}_{n}\right)$ are well bounded from below (see right image in figure 3.1). In this case, truncating (3.10) leads to a stable inversion formula. In particular, for $r_{\text {det }}=0$ one obtains

$$
\mathbf{F}_{z}\left\{F_{\sigma}\right\}(k, r)=\frac{2}{\pi^{2} r_{1}^{2}} \sum_{n \in \mathbb{N}} \frac{\mathbf{C}_{t}\left\{\mathbf{F}_{z}\left\{G_{\sigma}\right\}\right\}\left(k, \sqrt{k^{2}+\tilde{v}_{n}^{2}}\right)}{\sqrt{k^{2}+\tilde{v}_{n}^{2}}} \frac{J_{0}\left(r \tilde{v}_{n}\right)}{J_{1}\left(r_{1} \tilde{v}_{n}\right)^{2}}
$$

for any $r_{1} \geq 2 R$. Together with (2.10) this provides a novel inversion formula for PAT using point-like detectors on a cylindrical recording surface.

\section{Numerical experiments}

In practice one deals with discrete measurement data

$$
\mathrm{G}_{1}[\mathrm{~m}, \mathrm{n}]:=G_{\sigma_{1}}\left(z_{\mathrm{m}}, t_{\mathrm{n}}\right), \quad(\mathrm{l}, \mathrm{m}, \mathrm{n}) \in\left\{1, \ldots, \mathrm{N}_{\sigma}\right\} \times\left\{1, \ldots, \mathrm{N}_{z}\right\} \times\left\{1, \ldots, \mathrm{N}_{t}\right\},
$$

where $G_{\sigma}$ is as in (2.6), and where $\sigma_{1}=2 \pi(1-1) / \mathrm{N}_{\sigma}, z_{\mathrm{m}}=H(\mathrm{~m}-1) / \mathrm{N}_{z}$ and $t_{\mathrm{n}}=$ $T(\mathrm{n}-1) / \mathrm{N}_{t}$ are discrete samples of the angle, height and time, respectively. Here $H>0$ represents the finite height of the stack of circular integrating detectors (see figure 1.1) and $T$ is such that $G_{\sigma}(z, t)=0$ for $t \geq T$ and $z \in[0, H]$.

In this section we outline how to implement (3.1) and (3.5) in order to find an approximation

$$
\mathrm{F}_{1}[\mathrm{~m}, \mathrm{n}] \simeq F_{\sigma_{1}}\left(z_{\mathrm{m}}, r_{\mathrm{n}}\right), \quad(\mathrm{m}, \mathrm{n}) \in\left\{1, \ldots, \mathrm{N}_{z}\right\} \times\left\{1, \ldots, \mathrm{N}_{r}\right\}
$$

with $r_{\mathrm{n}}=r_{\operatorname{det}}(\mathrm{n}-1) / \mathrm{N}_{r}$. Having calculated such an approximation, one can reconstruct a discrete approximation to $f$ by applying the filtered back-projection algorithm of [8] for fixed m, see Remark 2.2.

A numerical reconstruction method based on (3.1) is as follows:

(i) The discrete Fourier transform (with respect to the first component) of the data

$$
\mathrm{F}\left\{\mathrm{G}_{1}\right\}[\mathrm{m}, \mathrm{n}]:=\sum_{\mathrm{m}^{\prime}=1}^{\mathrm{N}_{z}} \mathrm{G}_{1}\left[\mathrm{~m}^{\prime}, \mathrm{n}\right] e^{-i 2 \pi \mathrm{m}\left(\mathrm{m}^{\prime}-1\right) / \mathrm{N}_{z}}
$$

with $(\mathrm{m}, \mathrm{n}) \in\left\{-\mathrm{N}_{z} / 2, \ldots, \mathrm{N}_{z} / 2-1\right\} \times\left\{1, \ldots, \mathrm{N}_{t}\right\}$, is considered as an approximation to $\mathbf{F}\left\{F_{\sigma_{1}}\right\}\left(2 \pi \mathrm{m} / H, t_{\mathrm{n}}\right)$. 

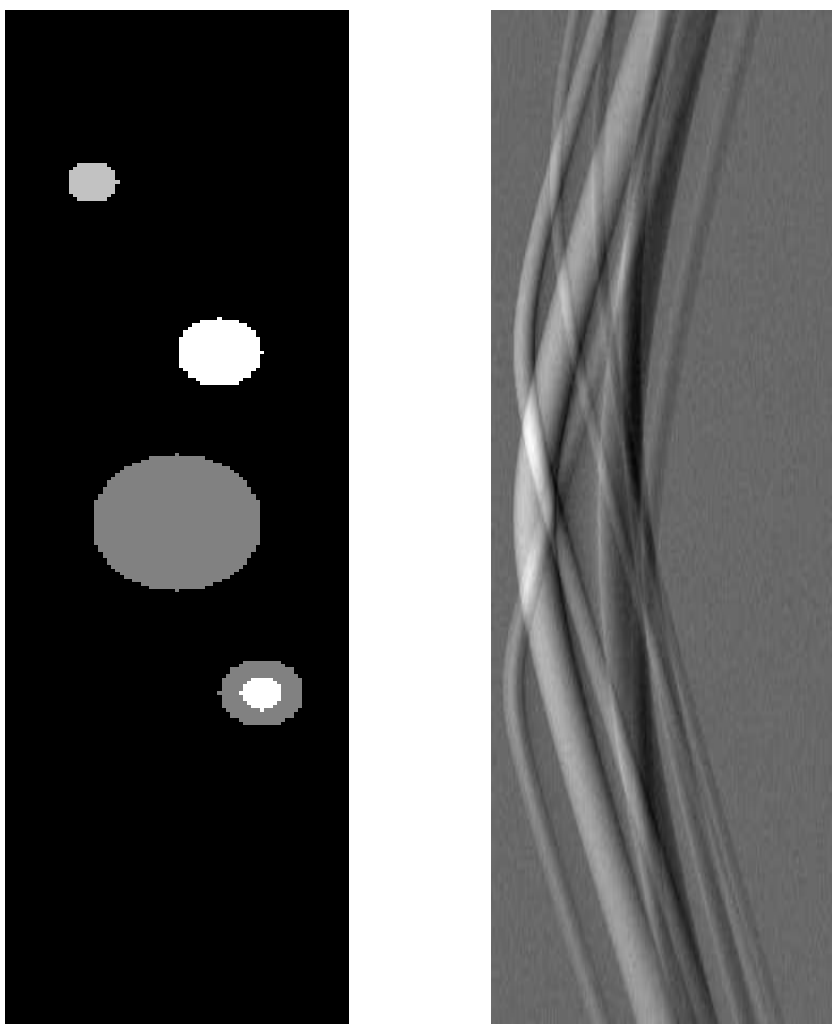

FIG. 4.1. Left: Cross section of five absorbing spheres ( $z$ versus $r$ ). Right: The measurement data with $10 \%$ Gaussian noise added ( $z$ versus $t$ ).

(ii) The sine transform $\mathbf{S}\left\{t \mathbf{F}\left\{F_{\sigma_{1}}\right\}\right\}$, evaluated at

$$
\omega_{\mathrm{m}, \mathrm{n}}=\sqrt{(2 \pi \mathrm{m} / H)^{2}+v_{\mathrm{n}}^{2}},(\mathrm{~m}, \mathrm{n}) \in\left\{-\mathrm{N}_{z} / 2, \ldots, \mathrm{N}_{z} / 2-1\right\} \times\left\{0, \ldots, \mathrm{N}_{r}-1\right\}
$$

is approximated by the trapezoidal rule, leading to

$$
\mathrm{S}\left\{\mathbf{t} F\left\{\mathrm{G}_{1}\right\}\right\}[\mathrm{m}, \mathrm{n}]:=\sum_{\mathrm{n}^{\prime}=1}^{\mathrm{N}_{t}} t_{\mathrm{n}^{\prime}} \mathrm{F}\left\{\mathrm{G}_{1}\right\}\left[\mathrm{m}, \mathrm{n}^{\prime}\right] \sin \left(\omega_{\mathrm{m}, \mathrm{n}} t_{\mathrm{n}^{\prime}}\right) .
$$

(iii) Finally, truncating the Fourier Bessel Series and approximating the inverse Fourier transform with the trapezoidal rule leads to discrete version of (3.1):

$$
\mathrm{F}_{1}[\mathrm{~m}, \mathrm{n}]:=\frac{4 T}{\pi r_{\operatorname{det}}^{3} \mathrm{~N}_{t}} \sum_{\mathrm{m}^{\prime}=-\mathrm{N}_{z}}^{\mathrm{N}_{z} / 2-1} \sum_{\mathrm{n}^{\prime}=0}^{\mathrm{N}_{\mathrm{r}}-1} \frac{v_{\mathrm{n}^{\prime}} \mathrm{S}\left\{\mathbf{t} \mathbf{F}\left\{\mathrm{G}_{1}\right\}\right\}\left[\mathrm{m}^{\prime}, \mathrm{n}^{\prime}\right]}{\omega_{\mathrm{m}^{\prime}, \mathrm{n}^{\prime}}^{2} J_{1}\left(r_{\operatorname{det}} v_{\mathrm{n}^{\prime}}\right)^{3}} J_{0}\left(r_{\mathrm{n}} v_{\mathrm{n}^{\prime}}\right) e^{-i 2 \pi \mathrm{m}^{\prime}(\mathrm{m}-1) / \mathrm{N}_{z}},
$$

with $(\mathrm{m}, \mathrm{n}) \in\left\{-\mathrm{N}_{z} / 2, \ldots, \mathrm{N}_{z} / 2-1\right\} \times\left\{0, \ldots, \mathrm{N}_{r}-1\right\}$ in formula (4.3).

A numerical reconstruction method using (3.1) can be obtained in an analogous 

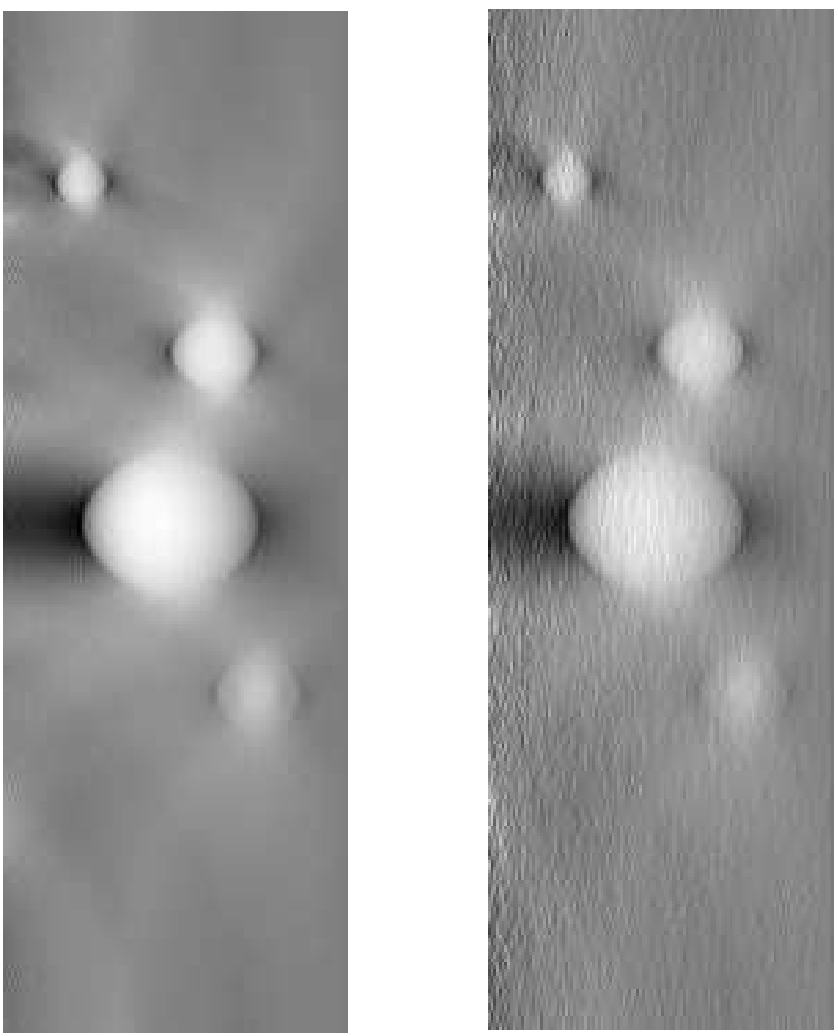

FIG. 4.2. Reconstruction with (3.1) from simulated (left) and noisy data (right).

manner. In this case one replaces (3.5) by

$$
\mathrm{F}_{1}[\mathrm{~m}, \mathrm{n}]:=\frac{4 T}{\pi r_{\operatorname{det}}^{2} \mathrm{~N}_{t}} \sum_{\mathrm{m}^{\prime}=-\mathrm{N}_{z}}^{\mathrm{N}_{z} / 2-1} \sum_{\mathrm{n}^{\prime}=0}^{\mathrm{N}_{r}-1} \frac{\mathrm{F}\left\{\mathrm{F}\left\{\mathrm{G}_{1}\right\}\right\}\left[\mathrm{m}^{\prime}, \mathrm{n}^{\prime}\right]}{\omega_{\mathrm{m}^{\prime}, \mathrm{n}^{\prime}} H_{0}^{(2)}\left(r_{\operatorname{det}} v_{\mathrm{n}^{\prime}}\right) J_{0}\left(r_{\operatorname{det}} v_{\mathrm{n}^{\prime}}\right)^{2}} J_{0}\left(r_{\mathrm{n}} v_{\mathrm{n}^{\prime}}\right) e^{-i 2 \pi \mathrm{m}^{\prime}(\mathrm{m}-1) / \mathrm{N}_{z}},
$$

which is the discrete analogue of (3.5).

To give a rough estimate of the computational complexity for the previous calculations let us assume $\mathrm{N}_{z}=\mathrm{N}_{r}=\mathrm{N}_{t}=\mathrm{N}_{\sigma}=$ : $\mathrm{N}$ and that the values of the sine function and the Bessel function are pre-computed and stored in lookup tables. Then the evaluation (4.1) needs $\mathcal{O}\left(\mathrm{N}^{2} \log \mathrm{N}\right)$ floating point operations (FLOPS) whereas (4.2) and (4.3) require $\mathcal{O}\left(\mathrm{N}^{3}\right)$ FLOPS. The filtered back projection formula (2.10) also requires $\mathcal{O}\left(\mathrm{N}^{3}\right)$ FLOPS, see [8]. For three dimensional reconstruction (4.1), (4.2), (4.3) and the filtered back-projection formula have to be applied $\mathrm{N}$ times. Hence the total number of FLOPS is estimated as

$$
\mathrm{N}_{\mathrm{FLOPS}}=\mathrm{N}\left(\mathcal{O}\left(\mathrm{N}^{2} \log \mathrm{N}\right)+\mathcal{O}\left(\mathrm{N}^{3}\right)+\mathcal{O}\left(\mathrm{N}^{3}\right)\right)=\mathcal{O}\left(\mathrm{N}^{4}\right) .
$$

Note that three dimensional back-projection type formulas which use point measurement data have complexity $\mathcal{O}\left(\mathrm{N}^{5}\right)$.

In the following numerical experiments we take $R=0.4, H=3.75$ and $T=4$. The synthetic initial data $f$ is assumed to be a superposition of radially symmetric 

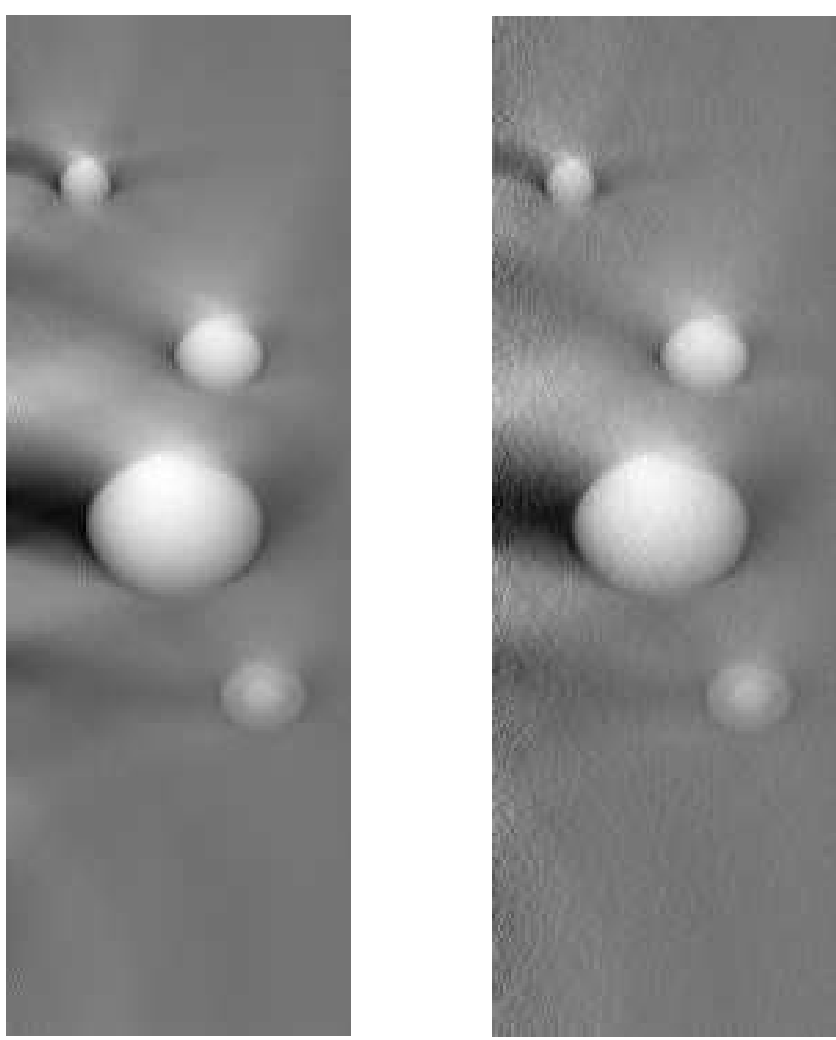

FIG. 4.3. Reconstruction with (3.5) from simulated (left) and noisy data (right).

objects around centers $\mathbf{x}_{n}$, i.e.,

$$
f(\mathbf{x})=\sum_{n} f_{n}\left(\left\|\mathbf{x}-\mathbf{x}_{n}\right\|\right), \quad \mathbf{x} \in \mathbb{R}^{3} .
$$

The acoustic pressure generated by a single radially symmetric object at position $\mathbf{x}$ and time $t$ is given by (see [13])

$$
p_{n}(\mathbf{x}, t)=\frac{\left\|\mathbf{x}-\mathbf{x}_{n}\right\|-t}{2\left\|\mathbf{x}-\mathbf{x}_{n}\right\|} f_{n}\left(\left|\left\|\mathbf{x}-\mathbf{x}_{n}\right\|-t\right|\right) .
$$

By the superposition principle the total pressure is

$$
p(\mathbf{x}, t)=\sum_{n=1}^{N} p_{n}(\mathbf{x}, t), \quad(\mathbf{x}, t) \in \mathbb{R}^{3} \times(0, \infty) .
$$

The measurement data $G_{\sigma}(z, t)=1 /(2 \pi) \int_{0}^{2 \pi} p\left(\Phi_{\sigma}\left(z, r_{\text {det }}, \alpha\right), t\right) d \alpha$, see (2.4), (2.6), were generated by evaluating of (4.6) followed by numerical integration over $\alpha$. The radius $r_{\text {det }}$ of the circular integrating detectors is chosen to be $2 R$. In this case the stack of circular integrating detectors fully encloses the synthetic initial data $f$, see right image in in figure 2.1. 
Figure 4.1 shows a vertical cross section of the initial pressure $f$ and the measurement data $G_{\sigma}$ where Gaussian noise with a variance of $10 \%$ of the maximal data valued is added. The stack of circular integrating detectors is centered to the left of the objects. In the presented discrete implementation $\mathbb{N}_{t}=320$ measurements in time and $\mathrm{N}_{z}=300$ in space are used. In both reconstructions the value $\mathrm{N}_{r}$ was chosen to be $\mathrm{N}_{r}=130$

The reconstructions of $F_{\sigma}$ with (4.3) from exact and noisy data are depicted in figure 4.2 from formula (4.3) and with formula (4.4) in figure 4.3. In the reconstructed images one notices some blurred boundaries which are limited data artifacts [20, 24, 29] arising from the finite height of the stack of circular integrating detectors. Moreover the images reconstructed with (4.4) are less sensitive to noise.

\section{Conclusion}

In this article a novel experimental buildup for PAT using circular integrating detectors was proposed. For collecting measurement data a fiber-based Mach-Zehnder or Fabry-Perot interferometer can be used as an circular integrating detector. We showed that the 3D imaging problem reduces to a series of 2D problems. This decomposition can be used to reduce the operation count of derived reconstruction algorithms. We derived two stable exact reconstruction formulas, (3.1) and (3.5), for the case that the object is contained in the stack of detecting circles. In the case where the object is outside the detecting circles, a stable reconstruction formula is obtained for the limiting case $r_{\text {det }} \ll R$. As a byproduct, this leads to a novel reconstruction formula (3.11) for PAT using point detectors on a cylindrical surface.

Acknowledgement. This work has been supported by the Austrian Science Foundation (FWF) within the framework of the NFN "Photoacoustic Imaging in Biology and Medicine", Project S10505-N20. Moreover, the work of M. Haltmeier has been supported by the technology transfer office of the University Innsbruck (transIT).

\section{REFERENCES}

[1] M. Abramowitz and I.A. Stegun, Handbook of Mathematical Functions, Dover, New York, 1972.

[2] M.L. Agranovsky, K. Kuchment and E.T. Quinto, Range descriptions for the spherical mean Radon transform, J. Funct. Anal., 248(2), 344-386, 2007.

[3] P. Burgholzer, C. Hofer, G. Paltauf, M. Haltmeier and O. Scherzer, Thermoacoustic tomography with integrating area and line detectors, IEEE Trans. Ultrason. Ferroeletr. Freq. Control, 52(9), 1577-1583, 2005.

[4] P. Burgholzer, G.J. Matt, M. Haltmeier and G. Paltauf, Exact and approximate imaging methods for photoacoustic tomography using an arbitrary detection surface, Phys. Rev. E, 75(4), 046706, 2007.

[5] C. Clason and M. Klibanov, The quasi-reversibility method for thermoacoustic tomography in a heterogeneous medium, SIAM J. Sci. Comput., accepted, 2007.

[6] C. Depeursinge (ed.), Novel Optical Instrumentation for Biomedical Applications III, Proceedings of SPIE-OSA, 6631, 2007.

[7] R.O. Esenaliev, I.V. Larina, K.V. Larin, D.J. Deyo, M. Motamedi and D.S. Prough, Optoacoustic technique for noninvasive monitoring of blood oxygenation: a feasibility study, App. Opt., 41(22), 4722-4731, 2002.

[8] D. Finch, M. Haltmeier and Rakesh, Inversion of spherical means and the wave equation in even dimensions, SIAM J. Appl. Math., 68(2), 392-412, 2007.

[9] D. Finch and Rakesh, The spherical mean value operator with centers on a sphere, Inverse Probl., 23(6), 37-49, 2007.

[10] H. Grün, M. Haltmeier, G. Paltauf and P. Burgholzer, Photoacoustic tomography using a fiber based Fabry-Perot interferometer as an integrating line detector and image reconstruction by model-based time reversal method, in [6], 2007. 
[11] M. Haltmeier, O. Scherzer, P. Burgholzer, R. Nuster and G. Paltauf, Thermoacoustic tomography \& the circular Radon transform: exact inversion formula, Math. Models Methods Appl. Sci., 17(4), 635-655, 2007.

[12] M. Haltmeier, O. Scherzer, P. Burgholzer and G. Paltauf, Thermoacoustic imaging with large planar receivers, Inverse Probl., 20(5), 1663-1673, 2004.

[13] M. Haltmeier, T. Schuster and O. Scherzer, Filtered backprojection for thermoacoustic computed tomography in spherical geometry, Math. Methods Appl. Sci., 28(16), 1919-1937, 2005.

[14] Y. Hristova, P. Kuchment and L. Nguyen, Reconstruction and time reversal in thermoacoustic tomography in acoustically homogeneous and inhomogeneous media, Inverse Problems, 24(5), 055006(25), 2008.

[15] R.G.M. Kolkman, E. Hondebrink, W. Steenbergen and F.F.M. De Mul, In vivo photoacoustic imaging of blood vessels using an extreme-narrow aperture sensor, IEEE J. Sel. Topics Quantum Electron., 9(2), 343-346, 2003.

[16] R.A. Kruger, K.D. Miller, H.E. Reynolds, W.L. Kiser, D.R. Reinecke and G.A. Kruger, Breast cancer in vivo: contrast enhancement with thermoacoustic CT at $434 \mathrm{MHz}$-feasibility study, Radiology, 216(1), 279-283, 2000.

[17] P. Kuchment and L.A. Kunyansky, Mathematics of thermoacoustic and photoacoustic tomography, European J. Appl. Math., 19, 191-224, 2008.

[18] L.A. Kunyansky, Explicit inversion formulae for the spherical mean Radon transform, Inverse Probl., 23(1), 373-383, 2007.

[19] L.A. Kunyansky, A series solution and a fast algorithm for the inversion of the spherical mean radon transform, Inverse Probl., 23(6), S11-S20, 2007.

[20] A.K. Louis and E.T. Quinto, Local tomographic methods in sonar, in Surveys on solution methods for inverse problems, Springer, Vienna, 147-154, 2000.

[21] S. Manohar, A. Kharine, J.C.G. Van Hespen, W. Steenbergen and T.G. Van Leeuwen, The Twente Photoacoustic Mammoscope: system overview and performance, Physics in Medicine and Biology, 50(11), 2543-2557, 2005.

[22] G. Paltauf, R. Nuster, M. Haltmeier and P. Burgholzer, Thermoacoustic computed tomography using a Mach-Zehnder interferometer as acoustic line detector, App. Opt., 46, 3352-3358, 2007.

[23] S.K. Patch and O. Scherzer, Special section on photo- and thermoacoustic imaging, Inverse Probl., 23, S1-S122, 2007.

[24] E.T. Quinto, Singularities of the X-ray transform and limited data tomography in $\mathbf{R}^{2}$ and $\mathbf{R}^{3}$, SIAM J. Math. Anal., 24(5), 1215-1225, 1993.

[25] O. Scherzer, M. Grasmair, H. Grossauer, M. Haltmeier and F. Lenzen, Variational Methods in Imaging, Appl. Math. Sci., Springer, New York, 167, 2009.

[26] I.N. Sneddon, The Use of Integral Transforms, McGraw-Hill, New York, 1972.

[27] M. Xu and L.V. Wang, Analytic explanation of spatial resolution related to bandwidth and detector aperture size in thermoacoustic or photoacoustic reconstruction, Phys. Rev. E, 67(5), 0566051-05660515 (electronic), 2003.

[28] M. Xu and L.V. Wang, Photoacoustic imaging in biomedicine, Rev. Sci. Instruments, 77(4), 1-22, 2006. Article ID 041101.

[29] Y. Xu, L.V. Wang, G. Ambartsoumian and P. Kuchment, Reconstructions in limited-view thermoacoustic tomography, Med. Phys., 31(4), 724-733, 2004.

[30] Y. Xu, M. Xu and L.V. Wang, Exact frequency-domain reconstruction for thermoacoustic tomography-II: cylindrical geometry, IEEE Trans. Med. Imag., 21, 829-833, 2002.

[31] X. Yang and L.V. Wang, Ring-based ultrasonic virtual point detector with applications to photoacoustic tomography, Applies Physics Letters, 90(25), 251103, 2007.

[32] G. Zangerl, M. Haltmeier and O. Scherzer, Circular integrating detectors in photo and thermoacoustic tomography, Inverse Probl. Sci. Eng., 17(1), 133-142, 2009. 